\title{
Comparison of the effects of three cryoprotectants on the cryopreservation of mouse subcutaneous tissue under different conditions
}

\author{
SHENGCUN WANG ${ }^{1,2}$, XIA YUAN $^{1}$, JING ZHOU $^{1}$, JING JIN $^{1}$, QISHENG ZUO ${ }^{1 *}$ and BICHUN LI ${ }^{1 *}$ \\ ${ }^{1}$ College of Animal Science and Technology, Yangzhou University, Yangzhou, Jiangsu 225009; \\ ${ }^{2}$ Laboratory Animal Center, Nantong University, Nantong, Jiangsu 226000, P.R. China
}

Received March 11, 2020; Accepted July 1, 2020

DOI: $10.3892 / \mathrm{etm} .2020 .9076$

\begin{abstract}
The subcutaneous tissue of animals contains different cell types, and different cells have different requirements for cryopreservation. This establishes obstacles that need to be overcome in the clinical application of tissue preservation. In the present study, the effects of different freezing rates and various concentrations of cryoprotectants on the cryopreservation of subcutaneous tissue of mice were compared, and these results provided basic research data that can be used to explore the optimal cryopreservation method for tissue. The effects of three cryoprotectants, dimethyl sulfoxide, glycerinum and 1,2-propanediol, and their concentrations on the cryopreservation of subcutaneous tissue of mice were compared with slow and rapid freezing rates. The results revealed that under various cryopreservation conditions, the percentage of fibroblasts that grow from the tissue following slow cryopreservation (19.8\%) was significantly higher than that following rapid freezing $(6.7 \%)$ at osmotic equilibrium for 10-20 min $(\mathrm{P}<0.05)$. After 19 days of culture, under the conditions of slow freezing, with 10, 20 and $30 \%$ glycerinum as a cryoprotectant, respectively, fibroblasts grew from 26.0, 16.7 and $16.7 \%$ of the tissues, respectively. No fibroblasts were indicated in the tissue mass cultured in any other tissue blocks treated with cryopreservation solutions. Under the condition of rapid freezing, fibroblasts grew from 6.7 and $6.7 \%$ tissue blocks of $20 \%$ DMSO and $10 \%$ glycerinum, respectively, following 19 days of culture. No fibroblasts were identified in the tissue mass cultured in the other tissue blocks treated with
\end{abstract}

Correspondence to: Professor Bichun Li or Dr Qisheng Zuo, College of Animal Science and Technology, Yangzhou University, 48 Wenhui East Road, Yangzhou, Jiangsu 225009, P.R. China

E-mail:yubcli@yzu.edu.cn

E-mail: 006664@yzu.edu.cn

${ }^{*}$ Contributed equally

Key words: cryopreservation, cryoprotectant, subcutaneous tissue cryopreservation solutions, and no fibroblasts were identified in the tissue blocks without osmotic balance before freezing.

\section{Introduction}

The large-scale clinical application of tissue engineering is required to provide a large number of directly usable tissues; however, the supply of fresh tissue to meet the needs of patients receiving tissue transplantation is often insufficient (1). Therefore, the study of the cryopreservation of tissue and the establishment of tissue banks is important in order to expand the clinical application of tissue transplantation. The cryopreservation of tissue is also of great significance to animal cloning. At present, the culture of somatic cells in vitro is an important link in the process of animal cloning; however, the survival period of somatic cells in vitro is less than one year, and the probability of chromosome variation may be increased due to frequent passage (2). If cryopreservation technology is used, the tissues needed to obtain cells are first cryopreserved, and are then resuscitated and cultured when needed so that the cells in the tissue can survive and expand by culture. This technique helped to prevent the adverse effects of long-term cell culture on the tissues (3). With the development of cryopreservation medical technology, the technology of cryopreservation of cells has been developed in recent studies $(4,5)$. However, the evaluation of different tissue freezing technology has not been undertaken sufficiently. Zhu et al (6), demonstrated that the effect of blood perfusion on cryopreservation in large tissues using DMSO was slightly improved compared with over-all soaking, especially during the preservation of skin and subcutaneous tissue. Ruan et al (7), transplanted human ovarian tissue pieces into different parts of nude mice after frozen resuscitation, and the ovaries returned to normal. Osei-Bempong et al (8), indicated that the cryopreservation of limbal stem cells with relatively low cryoprotectant concentration (5\%) exhibits a beneficial effect during low-temperature eye banking. The aim of the present study was to compare the effects of various cryoprotectants and their concentrations on the function and activity of mouse subcutaneous tissue cryopreservation under the conditions of different osmotic equilibrium times and freezing rates. 


\section{Materials and methods}

Animals. The experimental mice used in the present study were 15 C57BL/6 female mice, 2 months old and weighing 18-22 g, which were obtained from the Laboratory Animal Center of Nantong University. The mice were raised in the animal feeding room at a temperature of $20-26^{\circ} \mathrm{C}$, the air cleanliness was grade 7 and the humidity was $40-70 \%$. With a light cycle of $12 / 12 \mathrm{~h}$ and free access to drink clean water and eat nutritious formula mice feed. All animal procedures were conducted in accordance with animal research ethics, and all animal experiments were conducted in accordance with animal research ethics and approved by the Experimental Animal Ethics Committee of Nantong University.

Tissue samples. Mice at 60 days old were selected and were euthanized by cervical dislocation. Their body surface was sterilized with $75 \%$ alcohol. The abdominal skin was cut open, turned upside down and the subcutaneous tissue was removed. The tissue was washed with phosphate-buffered saline (PBS) at room temperature 4-5 times and the tissue was cut into small sections of $1 \mathrm{~mm}^{3}$ in size.

Freezing method. The subcutaneous tissues of mice were cut into sections of $1 \mathrm{~mm}^{3}$ in size and then placed into aseptic freezing tubes, with 3-5 sections per tube and with various cryoprotective solutions $(0.5 \mathrm{ml})$ at $\sim 23^{\circ} \mathrm{C}$. The cryopreservation was carried out by slow and rapid freezing, osmotic balance for 10-20 min and non-osmotic balance, 10 times. For slow freezing, the cryoprotective solution containing the tissue block was divided into two groups: One with osmotic equilibrium for 10-20 min and another with impermeable equilibrium at room temperature. The freezing tube was placed on the scaffold and treated on the surface of $\mathrm{CO}_{2}$ dry ice until the solution was frozen, and then stored in a freezer at $-80^{\circ} \mathrm{C}$. For rapid freezing, the cryoprotective solution containing the tissue blocks was divided into two groups: One with osmotic equilibrium group (10-20 min) and another with impermeable equilibrium group (non-osmotic equilibrium group). The solution was directly frozen in liquid nitrogen and then stored in a freezer at $-80^{\circ} \mathrm{C}$ for five days. The compositions of the different cryopreservation solutions are presented in Table I.

Tissue culture in vitro and detection of tissue growth. The freezing tube was taken out from the freezer at $-80^{\circ} \mathrm{C}$ and rapidly placed in a water bath at $37^{\circ} \mathrm{C}$. The frozen tissue was thawed at $37^{\circ} \mathrm{C}$ completely in $1 \mathrm{~min}$ in the water bath. Subsequently, the frozen solution was poured into a Petri dish together with the tissue. The tissue was then picked out with a syringe needle, washed in culture medium pre-heated at $37^{\circ} \mathrm{C} 4-5$ times, implanted into a six-well culture plate and cultured with $2 \mathrm{ml}$ solution. The culture conditions were as follows: DMEM was used as the basic culture medium (Sigma-Aldrich; Merck KGaA), supplemented with 10\% FBS (Sigma-Aldrich; Merck KGaA), $1 \%$ non-essential amino acid (Sigma-Aldrich; Merck KGaA) and $100 \mu \mathrm{g} / \mathrm{ml}$ penicillin and streptomycin (Sigma-Aldrich; Merck KGaA), with a culture temperature of $37.5^{\circ} \mathrm{C}$, saturated humidity and a $5 \% \mathrm{CO}_{2}$ concentration. Under a fluorescence microscope, the growth of the tissue was detected using Hoechst 33342 fluorescence staining (Beijing Reagan Biotechnology Co., Ltd.), at $1 \mu \mathrm{g} / \mathrm{ml}$, dyed at room temperature for $5 \mathrm{~min}$. The growth rate of the tissue was calculated using the following equation: Growth rate $=$ number of tissue blocks on which fibroblasts have grown after culture/total number of tissue blocks cultured after resuscitation $\mathrm{x} 100 \%$.

Statistical analysis. Statistical analysis was performed using SPSS11.5 (SPSS, Inc.) statistical software and a t-test was used to analyze the differences between groups. $\mathrm{P}<0.05$ was considered to indicate a statistically significant difference.

\section{Results}

Cell growth pattern. The growth pattern of the cells from the tissues is illustrated in Figs. 1 and 2. Numerous cells can be seen growing from the tissue sample.

Effect of slow freezing method on the cryopreservation of subcutaneous tissue. As shown in Table II, using slow freezing in various cryopreservation solutions, osmotic equilibrium for 10-20 min, subcutaneous tissue blocks of mice were cultured for 19 days, in different concentrations of cryoprotective liquid glycerinum. Fibroblasts were observed to of grown in tissue blocks of 10, 20 and $30 \%$ liquid glycerinum at 26.0, 16.7 and $16.7 \%$, respectively. No fibroblasts were identified under the condition of cryopreservation with DMSO and 1,2-propanediol. No fibroblasts were indicated in solution X and solution XI with both 1,2-propanediol and glycerinum. As also presented in Table II, there was no fibroblast growth in the tissue blocks directly frozen without osmotic balance in all cryopreservation solutions.

Effect of rapid freezing on the cryopreservation of mouse subcutaneous tissue. As presented in Table III, under the condition of rapid freezing, osmotic equilibrium for 10-20 min, subcutaneous tissue block culture for 19 days, freezing with 20\% DMSO and $10 \%$ glycerinum, and following culture in solution II and IV, 6.7 and $6.7 \%$ of the tissue blocks grew fibroblasts, respectively. Under the condition of other concentrations of cryopreservation solution such as solution I, III, V, VI, VII, VIII, IX, X and control solution, no fibroblasts were identified following culture. When the tissue blocks were directly frozen without osmotic balance, no fibroblasts were found in any of the types of cryoprotective solution following in vitro culture. When comparing the results presented in Tables II and III, it can be seen that when glycerinum was used as a cryoprotectant independently, the tissue blocks were frozen after osmotic equilibrium for 10-20 min. In solution IV, V and VI, the average growth rate with slow cryopreservation (19.8\%) was significantly higher than that with rapid cryopreservation (2.23\%). However, when DMSO was used as a cryoprotectant in solution II, the tissue growth rate with rapid cryopreservation $(6.7 \%)$ was significantly higher than that with slow cryopreservation $(0 \%)$ under the condition of osmotic equilibrium for 10-20 min (Tables II and III). This suggests that different cryoprotectants are required for different freezing rates.

\section{Discussion}

For the cryopreservation of cells or tissues, the addition of a certain amount of cryopreservation agent and selecting 
Table I. The composition of the cryopreservation solutions.

\begin{tabular}{|c|c|c|c|c|c|}
\hline Cryopreservation solutions & $\begin{array}{l}\text { DMSO } \\
(\%)\end{array}$ & $\begin{array}{c}\text { Glycerinum } \\
(\%)\end{array}$ & $\begin{array}{c}1,2-\text { propanediol } \\
(\%)\end{array}$ & $\begin{array}{l}\text { Fetal bovine serum } \\
\qquad(\%)\end{array}$ & $\begin{array}{c}\text { DMEM } \\
(\%)\end{array}$ \\
\hline Solution I & 10 & 0 & 0 & 10 & 80 \\
\hline Solution II & 20 & 0 & 0 & 10 & 70 \\
\hline Solution III & 30 & 0 & 0 & 10 & 60 \\
\hline Solution IV & 0 & 10 & 0 & 10 & 80 \\
\hline Solution V & 0 & 20 & 0 & 10 & 70 \\
\hline Solution VI & 0 & 30 & 0 & 10 & 60 \\
\hline Solution VII & 0 & 0 & 10 & 10 & 80 \\
\hline Solution VIII & 0 & 0 & 20 & 10 & 70 \\
\hline Solution IX & 0 & 0 & 30 & 10 & 60 \\
\hline Solution X & 0 & 10 & 10 & 10 & 70 \\
\hline Solution XI & 0 & 25 & 25 & 10 & 40 \\
\hline Control group & 0 & 0 & 0 & 10 & 90 \\
\hline
\end{tabular}

Table II. The growth rate of the frozen-thawed tissue with slow freezing.

\begin{tabular}{lccc}
\hline Groups & Osmotic equilibrium for $10-20$ min $(\%)$ & Without osmotic equilibrium $(\%)$ & P-value \\
\hline Solution III & 0.0 & 0.0 & - \\
Solution II & 0.0 & 0.0 & - \\
Solution III & 0.0 & 0.0 & - \\
Solution IV & 26.0 & 0.0 & P \\
Solution V & 16.7 & 0.001 \\
Solution VI & 16.7 & 0.0 & P $<0.001$ \\
Solution VII & 0.0 & 0.0 & P<0.001 \\
Solution VIII & 0.0 & 0.0 & - \\
Solution IX & 0.0 & 0.0 & - \\
Solution X & 0.0 & 0.0 & - \\
Solution XI & 0.0 & 0.0 & - \\
Control group & 0.0 & & - \\
\hline
\end{tabular}

-, no analysis performed.

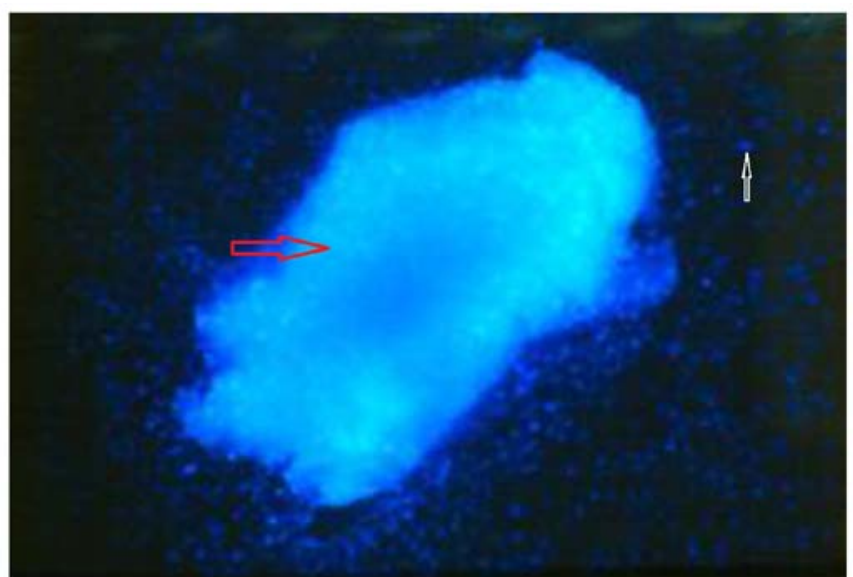

Figure 1. Cells after the tissue was frozen slowly in Solution IV and thawed. The small light blue spots indicate cells. The blue light in the center of the image indicates the tissue. The red arrows points to the tissue and the white arrow points to one of the derived cells (magnification, $\mathrm{x} 40$ ). the appropriate freezing rate is an important measure to implement to improve the cryopreservation effect on cells or tissues (9). The cryopreservation of stem cells and embryos has been successful $(10,11)$. The cryopreservation of rat testicular tissue (12), rat, pig and human islet tissue (13-16) and liver tissues of mice, rats, dogs, monkeys and humans has also been successful (17). Cryopreservation is not only conducive to the accumulation of donor tissue, but also significantly reduces its immunity (18). It has been demonstrated that the survival time of cryopreserved allogeneic tissue transplantation is significantly longer than that of fresh tissue transplantation (19), which may be due to the change in the immunogenicity of the skin following cryopreservation. However, the specific underlying mechanisms remain unclear (20).

The results of the present study revealed that with osmotic equilibrium for 10-20 min before freezing, the growth of the tissue blocks after tissue culture was as follows: When frozen using the slow freezing method, in the three different 
Table III. The growth rate of the frozen-thawed tissue with fast freezing.

\begin{tabular}{lccc}
\hline Groups & Osmotic equilibrium for 10-20 min (\%) & Without osmotic equilibrium (\%) & P-value \\
\hline Solution I & 0.0 & 0.0 & - \\
Solution II & 6.7 & 0.0 & P $<0.001$ \\
Solution III & 0.0 & 0.0 & - \\
Solution IV & 6.7 & 0.0 & $\mathrm{P}<0.001$ \\
Solution V & 0.0 & 0.0 & - \\
Solution VI & 0.0 & 0.0 & - \\
Solution VII & 0.0 & 0.0 & - \\
Solution VIII & 0.0 & 0.0 & - \\
Solution IX & 0.0 & 0.0 & - \\
Solution X & 0.0 & 0.0 & - \\
Solution XI & 0.0 & 0.0 & - \\
Control group & 0.0 & 0.0 & \\
\hline
\end{tabular}

,- no analysis performed.

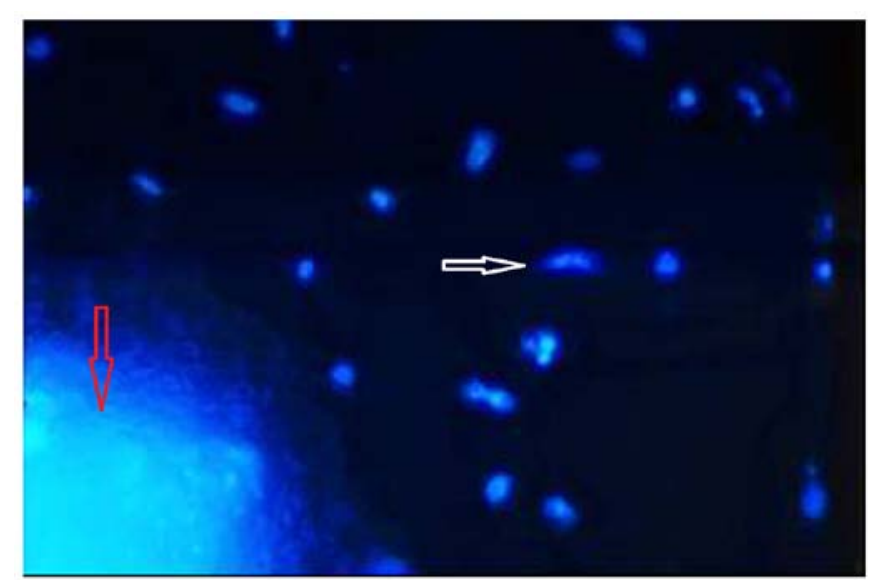

Figure 2. Tissue after being rapidly frozen in Solution IV and thawed. The small spots emitting light blue light indicate the cells. The blue light at the corner of the image is the tissue. The red arrows points to the tissue and the white arrow points to one of the cells (magnification, $\mathrm{x} 100$ ).

concentrations of cryopreservation liquid including glycerinum (10, 20 and 30\%), fibroblasts were grown in tissue blocks at 26.0, 16.7 and $16.7 \%$, respectively. No fibroblasts were found under the condition of cryopreservation with DMSO and 1,2-propanediol. When frozen using the rapid freezing method, only the tissue blocks in the solution containing $20 \%$ DMSO cryopreservation solution and $10 \%$ glycerinum cryopreservation solution survived following culture in vitro, and $6.7 \%$ of both tissue blocks grew fibroblasts. No fibroblasts were identified in the other cryopreservation solutions following culture in vitro. These experimental results indicated that the preservation effect of slow freezing cryopreservation is more effective than that of rapid freezing cryopreservation, which may be due to the fact that the cryopreservation solution can infiltrate into the tissue blocks when rapid freezing is used. However, it cannot fully enter the cell and combine with water. When the cell is frozen and cooled, the water in the extracellular solution freezes first and forms a hypertonic environment outside the cell, which causes the water in the cell to permeate outwards (21). However, due to the limited permeability per unit time of the cell membrane, the water molecules may not have had time to exudate, resulting in the formation of a large number of ice crystals in the intracellular solution, which damaged the cells, and finally resulted in no fibroblasts growing in the whole tissue block following culture. Previous studies results have revealed that the freezing rate had a significant effect on the viability of tissue blocks $(22,23)$. The results of the present study further confirmed this conclusion.

The results also demonstrated that the tissue growth rate with slow cryopreservation was significantly higher than that of rapid cryopreservation when glycerinum was used as a cryoprotectant and then frozen following osmotic equilibrium for 10-20 min. When DMSO was used as a cryoprotectant, the tissue growth rate with rapid cryopreservation $(6.7 \%)$ was significantly higher than that with slow freezing $(0 \%)$ under the condition of osmotic equilibrium for $10-20 \mathrm{~min}$. This result clearly suggests that glycerinum penetrates into tissue blocks at a slower rate than DMSO at the same time. When glycerinum is used as a cryopreservation solution, the slow freezing method was more effective, and when DMSO was used as a cryopreservation solution, the rapid freezing method was more suitable. If glycerinum is used as a cryopreservation solution to freeze tissue blocks by the rapid freezing method, the suitable concentration of glycerinum is $10 \%$. In the present study, when cryoprotectants were used in combination, no fibroblasts were found in the resuscitated tissue mass culture, which may be due to the fact that the combination of cryoprotectants used in the present study was not the optimal combination. Therefore, the protective effects of both cryoprotectants were not as prominent as those of each cryoprotectant alone, and this requires further study. In the present study, the effects of different freezing methods, different cryoprotectants and their concentrations on the cryoprotection of subcutaneous tissue of mice were compared. The growth characteristics of frozen subcutaneous tissue and the transplantation of frozen subcutaneous tissue requires further investigation in the future. 


\section{Acknowledgements}

Not applicable.

\section{Funding}

The present study was supported by the Key Research and Development Program (grant no. 2017YFE0108000), the National Natural Science Foundation of China (grant no. 31872341, 31572390); the Jiangsu Science and Technology Project (Youth Fund) (grant no. BK20180918); the Natural Science Research Project of Jiangsu Higher Education Institutions (grant no. 18KJB230008) and the High Level Talents Support Program of Yangzhou University (grant no. 11117).

\section{Availability of data and materials}

All data generated or analyzed during the present study are included in this published article.

\section{Authors' contributions}

BL and QZ conceived and designed the experiments. SW, $\mathrm{XY}$ and JZ performed the experiments. SW and JJ analyzed the data. SW and XY wrote the manuscript. SW, XY, JZ, JJ, BL and QZ edited the manuscript. All authors have read and approved the final manuscript.

\section{Ethics approval and consent to participate}

All animal procedures were conducted in accordance with animal research ethics, and all animal experiments are conducted in accordance with animal research ethics and approved by the Experimental Animal Ethics Committee of Nantong University (approval no. 20190507-002).

\section{Patient consent for publication}

Not applicable.

\section{Competing interests}

The authors declare that they have no competing interests.

\section{References}

1. HeydariZ,NajimiM,MirzaeiH,Shpichka A,Ruoss M,FarzanehZ, Montazeri L, Piryaei A, Timashev P, Gramignoli R, et al: Tissue engineering in liver regenerative medicine: Insights into novel translational technologies. Cells 9: 304, 2020.

2. Vcelar S, Melcher M, Auer N, Hrdina A, Puklowski A, Leisch F, Jadhav V, Wenger T, Baumann $\mathrm{M}$ and Borth $\mathrm{N}$ : Changes in chromosome counts and patterns in $\mathrm{CHO}$ cell lines upon generation of recombinant cell lines and subcloning. Biotechnol J 13: e1700495, 2018

3. Donfack NJ, Alves KA, Araújo VR, Cordova A, Figueiredo JR, Smitz J and Rodrigues APR: Expectations and limitations of ovarian tissue transplantation. Zygote 25: 391-403, 2017.

4. Dou M, Lu C, Sun Z and Rao W: Natural cryoprotectants combinations of 1-proline and trehalose for red blood cells cryopreservation. Cryobiology 91: 23-29, 2019.
5. Specchia C, Baggiani A, Immediata V, Ronchetti C, Cesana A, Smeraldi A, Scaravelli G and Levi-Setti PE: Oocyte cryopreservation in oncological patients: Eighteen years experience of a tertiary care referral center. Front Endocrinol (Lausanne) 10: 600, 2019.

6. Zhu Z, Qiao L, Liu F, He J, Rao X, Zhang S and Tang P: Dimethyl sulfoxide is less effective in immersing cryopreserved large pieces of tissue: A rabbit hind-limb model. Med Sci Monit 25: 7908-7913, 2019.

7. Ruan X, Cui Y, Du J, Jin J, Gu M, Chen S and Mueck AO: Randomized study to prove the quality of human ovarian tissue cryopreservation by xenotransplantation into mice. J Ovarian Res 12: 46, 2019.

8. Osei-Bempong C, Ghareeb AE, Lako M, Figueiredo FC and Armitage WJ: Defining the optimal cryoprotectant and concentration for cryopreservation of limbal stem cells. Cryobiology 84 : 98-102, 2018.

9. Hughes SM, Ferre AL, Yandura SE, Shetler C, Baker CAR, Calienes F, Levy CN, Astronomo RD, Shu Z, Lentz GM, et al: Cryopreservation of human mucosal tissues. PLoS One 13: e0200653, 2018.

10. Paynter JM, Chen J, Liu X and Nefzger CM: Propagation and Maintenance of Mouse Embryonic Stem Cells. Methods Mol Biol 1940: 33-45, 2019.

11. Cohen J, DeVane GW, Elsner CW, Fehilly CB, Kort HI, Massey JB and Turner TG Jr: Cryopreservation of zygotes and early cleaved human embryos. Fertil Steril 49: 283-289, 1988.

12. Benvenutti L, Salvador RA, Til D, Senn AP, Tames DR, Amaral NLL and Amaral VLL: Wistar rats immature testicular tissue vitrification and heterotopic grafting. JBRA Assist Reprod 22: 167-173, 2018.

13. Kojayan G, Whaley D, Alexander M, Rodriguez S, Lee S and Lakey JR: Improved cryopreservation yield of pancreatic islets using combination of lower dose permeable cryoprotective agents. Cryobiology 88: 23-28, 2019.

14. Nakayama K, Yamanaka T, Tamada Y, Hirabayashi M and Hochi S: Supplementary cryoprotective effect of carboxylated $\varepsilon$-poly-l-lysine during vitrification of rat pancreatic islets. Cryobiology 88: 70-74, 2019.

15. Powell-Palm MJ, Zhang Y, Aruda J and Rubinsky B: Isochoric conditions enable high subfreezing temperature pancreatic islet preservation without osmotic cryoprotective agents. Cryobiology 86: 130-133, 2019.

16. Kojayan GG, Alexander M, Imagawa DK and Lakey JRT: Systematic review of islet cryopreservation. Islets 10: 40-49, 2018.

17. Martignoni M, Monshouwer M, de Kanter R, Pezzetta D, Moscone A and Grossi P: Phase I and phase II metabolic activities are retained in liver slices from mouse, rat, dog, monkey and human after cryopreservation. Toxicol In Vitro 18: 121-128, 2004.

18. Berggren RB and Lether HB: Clinical use of viable frozen human skin. JAMA 194: 149-151, 1965.

19. Bondoc CC and Burke JF: Clinical Experiment with viable frozen human skin and a frozen skin bank. Ann Surg 174: 371-382, 1971.

20. Taylor MJ, London NJM, Thirdborough SM, Lake SP and James RFL: The cryobiology of rat and human dendritic cells: Preservation and destruction of membrane integrity by freezing. Cryobiology 27: 269-278, 1990.

21. Casula E, Asuni GP, Sogos V, Fadda S, Delogu F and Cincotti A: Osmotic behaviour of human mesenchymal stem cells: Implications for cryopreservation. PLoS One 12: e0184180, 2017.

22. Wise MH, Yates A, Gordon C and Johnson RWG: Subzero preservation of mechanically prepared porcine islets of Langerhans: Respone to a glucose challenge in vitro. Cryobiology 20: 211-218, 1983.

23. Hullett DA, Bethke KP, Landry AS, Leonard DK and Sollinger HW: Successful long-term cryopreservation and transplantation of human fetal pancreas. Diabetes 38: 448-453, 1989.

This work is licensed under a Creative Commons Attribution-NonCommercial-NoDerivatives 4.0 International (CC BY-NC-ND 4.0) License. 